BULL. AUSTRAL. MATH. SOC.

VOL. 28 (1983), 255-28I.

\title{
TWILLS WITH BOUNDED FLOAT LENGTH
}

\author{
Janet A. Hoskins, Cheryl E. Praeger \\ and Anne Penfold Street
}

\begin{abstract}
The simple twills on $n$ harnesses can be classified according to their maximum float length. The number of $n$-harness twills with specified maximum float length is determined both by Burnside enumeration and, for $n \leq 20$, by an adaptation of a sieve algorithm for twills.
\end{abstract}

\section{Introduction}

Every simple twill on $n$ harnesses corresponds to an equivalence class of cyclic binary sequences of length $n$, where two such sequences $S=\left(s_{1}, s_{2}, \ldots, s_{n}\right)$ and $T=\left(t_{1}, t_{2}, \ldots, t_{n}\right)$ are equivalent if and only if one can be transformed into the other by a shift $\left(s_{i}=t_{i+1}\right)$, by reversal $\left(s_{i}=t_{n+1-i}\right)$, by complementation $\left(s_{i}=\sim t_{i}\right)$, or by some finite sequence of these operations. (Subscripts are added modulo $n$. ) In other words, $S$ and $T$ are equivalent under the action of $D_{2 n} \times S_{2}$, the direct product of the dihedral group of order $2 n$ with the symmetric group of degree 2 .

Conversely, every equivalence class of cyclic binary sequences of

Received 5 July 1983. The authors thank the Department of Computer Science, University of Manitoba, and the Departments of Mathematics of the Universities of Queensland and Western Australia for the financial assistance which enabled them to collaborate. Anne Penfold Street also grate fully acknowledges support from ARGC.

Copyright Clearance Centre, Inc. Serial-fee code: 0004-9727/83 $\$ A 2.00+0.00$. 
length $n$ corresponds to a simple twill on $n$ harnesses, except for the all zero (or all one) sequence.

This correspondence between twills and sequences is explained in Grünbaum and Shephard [2], Hoskins [3] and Hoskins and Street [4], for example; see also the references cited in [4]. It has been used to determine the total number of twills on $n$ harnesses, both by Burnside enumeration [1, p. 191] and by a sieving algorithm, and to determine the number of twills on $n$ harnesses with certain special properties. A further case is now considered.

A sequence with $s_{i} \neq s_{i+1}=\ldots=s_{i+k} \neq s_{i+k+1}$ is said to have a float of length $k$, that is, a block of $k$ consecutive symbols which are equal. Since the maximum float length is an important property of a twill, the number of twills on $n$ harnesses with given maximum float length are determined here.

The maximum float length is obviously closely related to the number of breaks in the sequence, where $\left(s_{1}, s_{2}, \ldots, s_{n}\right)$ has $m$ breaks if and only if $s_{i} \neq s_{i+1}$ for precisely $m$ distinct values of $i=1, \ldots, m$. For example, the sequences 000111 and 00100111 both have maximum float length three, and have two and four breaks respectively. Note that the number of breaks must always be even.

In order to state our results, we introduce the following notation.

We denote by $F(n, k)$ the number of equivalence classes of binary sequences of length $n$, with maximum float length $k$, and by $F(n, k, m, x)$ the number of classes of such sequences with exactly $m$ breaks and exactly $x$ floats of length $k$. Then

$$
F(n, k)=\sum F(n, k, m, x),
$$

where the summation is over all $m$ and $x$ satisfying the following conditions :

(a) $m$ is even, and

$$
\begin{gathered}
\lceil n / k\rceil+\delta \leq m \leq n-k+\varepsilon, \\
\delta, \varepsilon=0 \text { or } 1, \delta \equiv\lceil n / k\rceil(\bmod 2), \varepsilon \equiv n-k(\bmod 2) ;
\end{gathered}
$$


(b) if $n=k q+r, 0 \leq r \leq k-1$, then

$$
1 \leq x \leq\left\{\begin{array}{rrll}
q & \text { if } r \geq 2, & \\
& \text { or if } r=1, q \text { odd, } \\
& \text { or if } r=0, q \text { even; } \\
q-1 & \text { if } r=1, q \text { even } \\
& \text { or if } r=0, q \text { odd; }
\end{array}\right.
$$

(c) $x \leq m \leq n-k x+x-\varepsilon, \varepsilon \equiv n-k x+x(\bmod 2), \varepsilon=0$ or 1 , and if $m=x$, then $k \mid n, n / k$ is even, and $x=n / k$.

Similarly, we denote by $F(n, k,-, x)$ the sum of $F(n, k, m, x)$ over all $m$ satisfying the conditions stated above, for given $x$.

Note that we assume $k<n$, for if $k=n$, then $m=0$, no corresponding twill exists, and condition (a) becomes $2 \leq m \leq 0$. Hence $F(n, n)$ is not defined.

Let $S(n, k, m, x)$ denote the set of binary sequences of length $n$, with maximum float length $k, m$ breaks, and $x$ floats of length $k$, and let $s \in S(n, k, m, x)$, where $s=\left(s_{1}, \ldots, s_{n}\right)$. We make the convention that $s_{n} \neq s_{1}$ (which is always possible since $k<n$ ), and we can associate with $s$ the sequence of positive integers

$$
r(s)=\left(r_{1}, \ldots, r_{m}\right),
$$

where $s_{1}=\ldots=s_{r_{1}} \neq s_{r_{1}+1}=\ldots=s_{r_{1}+r_{2}} \neq s_{r_{1}+r_{2}+1}=\ldots$. (In the traditional break notation of weaving, this would be written

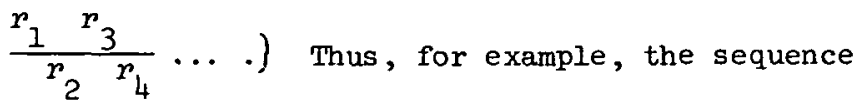

$$
s=(00101) \in S(5,2,4,1)
$$

has associated sequence

$$
r(s)=(2,1,1,1)
$$

Let $R(n, k, m, x)$ be the set of positive integer sequences of length $m$, where

$$
r=\left(r_{1}, \ldots, r_{m}\right) \in R(n, k, m, x)
$$


satisfies

$$
\begin{aligned}
& 1 \leqq r_{i} \leqq k, \text { for } i=1,2, \ldots, m, \\
& r_{i}=k \text { for exactly } x \text { values of } i,
\end{aligned}
$$

and

$$
\sum_{i=1}^{m} r_{i}=n \text {. }
$$

(Note that $r$ is actually a composition of $n$ into $m$ parts, where $x$ parts equal $k$, and $m-x$ parts are less than $k$.$) Then each$

$s \in S(n, k, m, x)$ corresponds to $r(s) \in R(n, k, m, x)$, and conversely each $r \in R(n, k, m, x)$ corresponds to exactly two sequences $\mathbf{S}, \mathbf{S}^{\prime} \in S(n, k, m, x)$, where one is the complement of the other. Thus these two sequences, $\mathbf{s}$ and $\mathbf{s}^{\prime}$, are equivalent in $S(n, k, m, x)$.

The equivalence relation on sequences in $S(n, k, m, x)$, induced by the action of $D_{2 n} \times S_{2}$, corresponds to an equivalence relation on $R(n, k, m, x)$, where two sequences in $R(n, k, m, x)$ are equivalent if and only if one can be transformed into the other by a cyclic shift, $\sigma$, by a reversal $\rho$, or by some finite sequence of these operations.

$$
\text { If } r=\left(r_{1}, r_{2}, \ldots, r_{m-1}, r_{m}\right) \text {, then } r o=\left(r_{m}, r_{1}, r_{2}, \ldots, r_{m-1}\right) \text {, }
$$

and $r \rho=\left(r_{m}, r_{m-1}, \ldots, r_{2}, r_{1}\right)$.

Hence the equivalence relation on $R(n, k, m, x)$ is induced by the dihedral group of order $2 m$ defined by

$$
G=\langle\sigma, \rho\rangle \text {. }
$$

Thus $F(n, k, m, x)$, the number of equivalence classes of binary sequences in $S(n, k, m, x)$ under the action of $D_{2 n} \times S_{2}$, equals the number of equivalence classes of positive integer sequences in $R(n, k, m, x)$ under the action of $G$.

Our counting arguments will involve $c(N, M, n)$, the number of compositions of $n$ into exactly $M$ parts, none exceeding $N$, and we recall that 


$$
c(N, M, n)=\sum\left(\begin{array}{c}
M \\
b_{1}, \ldots, b_{t}
\end{array}\right),
$$

where the surmation is over all partitions $I$ of $n$ into $b_{i}$ parts equal to $a_{i}, i=1, \ldots, t$, so that

$$
\Pi=a_{1}^{b_{1}} \ldots a_{t}^{b_{t}}
$$

where $\sum_{i=1}^{t} a_{i} b_{i}=n, \sum_{i=1}^{t} b_{i}=M, 1 \leqq a_{1} \leqq \ldots \leqq a_{t} \leqq N$.

We make the convention that

$$
c(N, 0, n)=\left\{\begin{array}{lll}
1 & \text { if } n=0, \\
0 & \text { if } n>0 .
\end{array}\right.
$$

Finally, we let $\phi$ denote Euler's phi function.

We are now ready to state our main result, evaluating $F(n, k, m, x)$ when $n, k, m, x$ are such that $F(n, k, m, x)>0$. (This rules out, for example, the case where $x$ is odd and $F(n, k, x, x)=0$.

THEOREM 1. (a) If $n=k x$, then $m=x=n / k$ is even, and

$$
F(n, k, n / k, n / k)=1 \text {. }
$$

(b) If $n>k x$, then $m>x$, and two cases arise:

(i) if $x$ is odd, then

( $\alpha$ ) either $m=x+1$, so that $n-k x<k$ and

$$
F(n, k, m, m-1)=1 \text {, }
$$

(B) or $m>x+1$ and

$$
\begin{aligned}
F(n, k, m, x)=\frac{1}{2 m} \sum_{d} \phi(d) & \cdot\left(\begin{array}{l}
m / d \\
x / d
\end{array}\right) \cdot c\left(k-1, \frac{m-x}{d}, \frac{n-k x}{d}\right) \\
& +\frac{1}{2}\left(\begin{array}{l}
(m-2) / 2 \\
(x-1) / 2
\end{array}\right) \underset{u}{\sum_{u} c\left(k-1, \frac{m-1-x}{2}, \frac{n-k x-u}{2}\right),}
\end{aligned}
$$

where the summations are (respectively) over all $d$ such that $d \operatorname{lgcd}(n, m, x)$ and all $u$ such that

$1 \leqq u \leqq \min (k-1, n-(k-1) x-m+1), u \equiv n-k x(\bmod 2) ;$ 
(ii) if $x$ is even, then

(a) either $m=x+2$, so that $n-k x \leq 2 k-2$ and $F(n, k, m, m-2)=\left\{\begin{array}{l}m(n-k x-1+\delta) / 4 \text { for } n-k x \leq k, \\ m(2 k-1+\delta-(n-k x)) / 4, \text { for } k \leq n-k x,\end{array}\right.$

(B) or $m>x+2$ and

$$
\begin{aligned}
F(n, k, m, x)=\frac{1}{2 m} \sum_{d} \phi(d) & \cdot\left(\begin{array}{l}
m / d \\
x / d
\end{array}\right) \cdot c\left(k-1, \frac{m-x}{d}, \frac{n-k x}{d}\right) \\
+ & \frac{1}{2}\left(\begin{array}{c}
(m-2) / 2 \\
x / 2
\end{array}\right) \sum_{u, v} c\left(k-1, \frac{m-x-2}{2}, \frac{n-k x-u-v}{2}\right) \\
& +\frac{1}{2} \delta\left(\begin{array}{l}
m / 2 \\
x / 2
\end{array}\right) c\left(k-1, \frac{m-x}{2}, \frac{n-k x}{2}\right),
\end{aligned}
$$

where $\delta=0$ or $1, \delta \equiv n-1(\bmod 2)$, and the summations are (respectively) over all $d$ such that $d \mid \operatorname{gcd}(n, m, x)$, and $a l l u, v$ such that $1 \leq u<v \leq k-1$, $u+v \leq n-(k-1) x-m+2, u+v \equiv n-k x(\bmod 2)$.

In certain cases, the statement of Theorem 1 can be greatly simplified. For example, if $x=1$, so that only one float of length $k$ occurs, then by Theorem 1 (b) (i), either $m=2$, and $F(n, k, 2,1)=1$ or $m \geqq 4$, and

$$
F(n, k, m, 1)=\frac{1}{2} c(k-1, m-1, n-k)+\frac{1}{2} \sum_{u} c\left(k-1, \frac{m-2}{2}, \frac{n-k-u}{2}\right),
$$

where the summation is over all $u$ such that $1 \leqq u \leqq \min (k-1, n-k-m+2)$, and $u \equiv n-k(\bmod 2)$.

In particular, if $k>n / 2$, then $x=1$, giving the following result.

THEOREM 2. If $n / 2<k \leqq n-3$, then

$$
F(n, k)=2^{n-k+3}+2^{\lfloor(n-k-3) / 2\rfloor} \text {. }
$$

Also $F(n, n-1)=F(n, n-2)=1$.

Again if $n / 3 \leqq k \leqq n / 2$, then $x=1$ or $x=2$. Considering the case $x=1$ first, we have the following result.

THEOREM 3. If $n / 3 \leqq k \leqq n / 2$, then

(a) either $n=2 k$ or $2 k+1$, and 


$$
F(n, k,-, 1)=2^{n-k-3}+2^{\lfloor(n-k-3) / 2\rfloor}-1 ;
$$

(B) or $n \geqq 2 k+2$, and

$$
\begin{aligned}
F(n, k,-, 1)=2^{n-k-3}+2^{\lfloor(n-k-3) / 2\rfloor}-2^{\lceil(n-2 k-3) / 2\rceil} & -2^{n-2 k-2}-(n-2 k) 2^{n-2 k-3} .
\end{aligned}
$$

Next, if $x=2$, so that only two floats of length $k$ occur, we may simplify Theorem 1 slightly differently. If $n=2 k$, then by part (a), we have $F(n, k, 2,2)=1=F(n, k,-, 2)$. Otherwise, we apply part (b) (ii), since $n>2 k$. Either $m=4$, so that in fact $n \geqq 2 k+2$, and $F(n, k, 4,2)=n-2 k-1+\delta$, or $m \geqq 6$, so that $n \geqq 2 k+4$, and

$$
\begin{aligned}
F(n, k, m, 2)= & \frac{1}{2 m}\left\{\left(\begin{array}{l}
m \\
-2
\end{array}\right) c(k-1, m-2, n-2 k)+\delta \cdot \frac{m}{2} c\left(k-1, \frac{m-2}{2}, \frac{n-2 k}{2}\right)\right\} \\
& +\frac{m-2}{4} \sum_{u, v} c\left(k-1, \frac{m-4}{2}, \frac{n-2 k-u-v}{2}\right)+\frac{\delta m}{4} \cdot c\left(k-1, \frac{m-2}{2}, \frac{n-2 k}{2}\right) \\
= & \frac{m-1}{4} \cdot c(k-1, m-2, n-2 k)+\frac{\delta(m+1)}{4} \cdot c\left(k-1, \frac{m-2}{2}, \frac{n-2 k}{2}\right) \\
& +\frac{m-2}{4} \sum_{u, v} c\left(k-1, \frac{m-4}{2}, \frac{n-2 k-u-v}{2}\right),
\end{aligned}
$$

where $\delta=0$ or $1, \delta \equiv n-1(\bmod 2)$, the summation is over all $u$ and $v$ such that $1 \leqq u<v \leqq k-1, u+v \leqq n-2 k+4-m$, and $u+v \equiv n-2 k(\bmod 2)$ and this term will occur only for $n \geqq 2 k+6$ and $m \leqq n-2 k+1$.

Now we can finish dealing with the range $n / 3 \leqq k \leqq n / 2$, with $x=2$.

THEOREM 4. If $n / 3 \leqq k \leqq n / 2$, then

$$
F(n, k,-, 2)=\left\{\begin{aligned}
1 & \text { if } n=2 k, \\
0 & \text { if } n=2 k+1, \\
2 & \text { if } n=2 k+2 \text { or } 2 k+3, \\
7 & \text { if } n=2 k+4, \\
10 & \text { if } n=2 k+5,
\end{aligned}\right.
$$

and if $n \geqq 2 k+6$, then

$$
\begin{aligned}
F(n, k,-, 2)=(n-2 k-1+\delta)+ & (n-2 k-1)\left(2^{n-2 k-5}-\frac{3}{4}\right)+2^{n-2 k-3} \\
& +\delta(n-2 k-2) 2^{(n-2 k-8) / 2}+\delta \cdot 5 \cdot 2^{(n-2 k-6) / 2} \\
& -\frac{5}{4} \delta+\left\lfloor\frac{n-2 k-1}{2}\right\rfloor \cdot 2^{\lfloor(n-2 k-5) / 2\rfloor}-\frac{1}{2}\left\lfloor\frac{n-2 k-1}{2}\right\rfloor,
\end{aligned}
$$


where $\delta=0$ or $1, \delta \equiv n-1(\bmod 2)$.

Theorems 2, 3 and 4 result from simplifying Theorem 1 for $n / k$ small, which forces all possible $x$ to be small. If $k$ is small instead, Theorem 1 also simplifies.

THEOREM 5. If $k=2$, then $E(n, 2, m, x)=0$ unless $n=m+x$, so that

$$
F(n, 2,-, x)=F(n, 2, n-x, x) .
$$

Moreover if $n=m+x$, then two cases arise:

(i) if $x$ is odd, then

(a) either $m=x+1$ and

$$
F(2 x+1,2, x+1, x)=1
$$

(B) or $m \geqq x+3$ and

$$
F(m+x, 2, m, x)=\frac{1}{2 m} \sum_{d} \phi(d) \cdot\left(\begin{array}{l}
m / d \\
x / d
\end{array}\right)+\frac{1}{2}\left(\begin{array}{l}
(m-2) / 2 \\
(x-1) / 2
\end{array}\right) ;
$$

(ii) if $x$ is even, then

(a) either $m=x$ and

$$
F(2 x, 2, x, x)=1
$$

(B) or $m=x+2$ and

$$
F(2 x+2,2, x+2, x)=(x+2) / 2
$$

( $\gamma$ ) or $m \geqq x+4$ and

$$
F(m+x, 2, m, x)=\frac{1}{2 m} \sum_{d} \phi(d)\left(\begin{array}{l}
m / d \\
x / d
\end{array}\right)+\frac{1}{2}\left(\begin{array}{l}
m / 2 \\
x / 2
\end{array}\right) .
$$

The summations in both cases are over all $d$ such that $d \mid \operatorname{gcd}(n, m, x)$.

THEOREM 6. If $k=3$ and $m>x$, two cases arise:

(i) if $x$ is odd, then

(a) either $m=x+1$, so that $n-3 x<3$ and

$$
F(n, 3, m, m-1)=1 \text {, }
$$

(B) or $m>x+1$ and

$$
\begin{gathered}
F(n, 3, m, x)=\frac{1}{2 m} \sum_{d} \phi(d) \cdot\left(\begin{array}{l}
m / d \\
x / d
\end{array}\right)\left(\begin{array}{c}
(m-x) / d \\
(n-2 x-m) / d
\end{array}\right)+\frac{1}{2}\left(\begin{array}{l}
(m-2) / 2 \\
(x-1) / 2
\end{array}\right)\left(\begin{array}{c}
(m-1-x) / 2 \\
(n-2 x-m+1-u) / 2
\end{array}\right) \\
\text { where } u=1 \text { or } 2, u \equiv n-x(\bmod 2) ;
\end{gathered}
$$

(ii) if $x$ is even, then

(a) either $m=x+2$, so that $n-3 x \leq 4$ and 


$$
F(n, 3, m, m-2)=m(n-3 x-1+\delta) / 4,
$$

(B) or $m>x+2$ and

$$
\begin{aligned}
F(n, 3, m, x)=\frac{1}{2 m} \sum_{d} \phi(d) & \left(\begin{array}{l}
m / d \\
x / d
\end{array}\right)\left(\begin{array}{c}
(m-x) / d \\
(n-m-2 x) / d
\end{array}\right) \\
& +\frac{1}{2} \varepsilon\left(\begin{array}{c}
(m-2) / 2 \\
x / 2
\end{array}\right)\left(\begin{array}{c}
(m-x-2) / 2 \\
(n-3 x-3) / 2
\end{array}\right)+\frac{1}{2} \delta\left(\begin{array}{l}
m / 2 \\
x / 2
\end{array}\right)\left(\begin{array}{c}
(m-x) / 2 \\
(n-m-2 x) / 2
\end{array}\right),
\end{aligned}
$$

where $\delta, \varepsilon=0$ or $1, \delta \equiv n-1(\bmod 2), \varepsilon \equiv n(\bmod 2)$. The summations in both cases are over all $d$ such that $d \mid \operatorname{gcd}(n, m, x)$.

Values of $F(n, k,-, x)$ for $n \leqq 20$, and $x=1$ to 10 , have been calculated by a sieving algorithm, based on that described in [4]. These values have been checked against the results of Theorems 1 to 6 . For $4 \leqq k<n / 3$, we have been unable to simplify the statement of Theorem 1 into any more convenient form. In checking $F(n, 3,-, x)$ from Theorem 6 , we must sum over $m$, as $m$ runs through all even numbers from $\lceil(n-x) / 2\rceil$ to $2\lceil(n-2 x-1) / 2\rceil$.

\section{Details of proofs}

We shall use several binomial identities, especially the following:

(B1) $\sum_{k=0}^{n}\left(\begin{array}{l}n \\ k\end{array}\right)=2^{n}$,

(B2) $\sum_{m=0}^{\lfloor n / 2\rfloor}\left(\begin{array}{c}n \\ 2 m\end{array}\right)=2^{n-1}$,

(B3) $\sum_{x=y}^{z}\left(\begin{array}{l}x \\ y\end{array}\right)=\left(\begin{array}{l}z+1 \\ y+1\end{array}\right)$,

(B4) (m-1) $\left(\begin{array}{c}n-2 k \\ m-2\end{array}\right)=(m-2)\left(\begin{array}{c}n-2 k \\ m-2\end{array}\right)+\left(\begin{array}{c}n-2 k \\ m-2\end{array}\right)=(n-2 k)\left(\begin{array}{c}n-2 k-1 \\ m-3\end{array}\right)+\left(\begin{array}{c}n-2 k \\ m-2\end{array}\right)$.

Proof of Theorem 1. Each r-sequence determines uniquely an (unordered) partition

$$
\pi=a_{1}^{b_{1}} \ldots a_{t}^{b_{t}}
$$

of $n-k x=\sum_{i=1}^{t} a_{i} b_{i}$ into $m-x=\sum_{i=1}^{t} b_{i}$ parts, where 
$1 \leqq a_{1}<a_{2}<\ldots<a_{t} \leqq k-1$, and $t \geqq 1 ;$ namely, $r$ has $b_{i}$ entries equal to $a_{i}$ for each $i=1, \ldots, t$. Conversely, any partition $\Pi$ of $n-k x$ into $m-x$ parts, each part at most $k-1$, corresponds to some $r$-sequences in $R(n, k, m, x)$, and the set $R(I I)$ of $r$-sequences corresponding to a given partition $\Pi$ is fixed setwise by the group $G$, defined in (1). If $n=k x$, then $m=x=n / k$. Either $m$ is even, and $F(n, k, n / k, n / k)=1$, or $m$ is odd and $F(n, k, n / k, n / k)=0$. We may now assume that $n>k x$, and hence that $m>x$.

Let $\operatorname{fix} g=\{r \in R(n, k, m, x) \mid r g=r\}$, for each $g \in G$. By Burnside's lemma, the number of G-orbits in $R(\Pi)$ is

$$
\frac{1}{2 m} \cdot \sum_{g \in G}|\mathrm{fix} g \cap R(\Pi)|
$$

Hence for each $g$ in $G$ we evaluate $\mid$ fix $g \cap R(I) \mid$ and sum over all partitions $\Pi$. We consider the various elements $g$ in $G$.

(a) Let $g=\sigma^{2}$, where $l \leqq l \leqq m$. Then $r g=r$ if and only if, for all $i$,

$$
r_{i}=r_{i+(m / d)},
$$

where $m / d=\operatorname{gcd}(2, m)$, and subscripts are to be taken modulo $m$, that is, $r$ consists of $d$ repetitions of a subsequence $r^{\prime}=\left(r_{1}, \ldots, r_{m / d}\right)$. Also, $x=d x^{\prime}, b_{i}=d b_{i}^{\prime}$ where $b_{i}^{\prime}$ is an integer, for $i=1,2, \ldots, t, \sum_{1 \leqq i \leqq m / d} r_{i}=n / d=n^{\prime}$, say, and the subsequence $r^{\prime}$ corresponds to a partition

(4) $\left\{\begin{array}{c}\Pi^{\prime}=a_{1}^{1} \ldots a_{t}^{b_{t}^{\prime}} \\ \text { of }(n-k x) / d=n^{\prime}-k x^{\prime}=\sum_{i=1}^{t} a_{i} b_{i}^{\prime} \text { into }(m-x) / d=m / d-x^{\prime}=\sum_{i=1}^{t} b_{i}^{\prime} \\ \text { parts where } 1 \leqq a_{1}<a_{2}<\ldots<a_{t} \leqq k-1, \text { and } t \geqq 1 .\end{array}\right.$ We note of course that $r^{\prime}$ determines $r$ uniquely. To determine $\mid$ fix $\sigma^{2} \mid$, we now sum over all partitions $\Pi$, bearing 
Twill with bounded float length

265

in mind that $d=m / \operatorname{gcd}(l, m)$. Two cases arise:

(i) $d \nmid \operatorname{ged}(n, x)$, and $\left|f i x \sigma^{2}\right|=0$;

(ii) $d \mid \operatorname{gcd}(n, x)$, and

$$
\mid \text { fix } \sigma^{2} \mid=\sum\left[\begin{array}{c}
m / d \\
b_{1}^{\prime}, \ldots, b_{t}^{\prime}, x / d
\end{array}\right]=\sum\left[\begin{array}{l}
m / d \\
x / d
\end{array}\right)\left(\begin{array}{c}
(m-x) / d \\
b_{1}^{\prime}, \ldots, b_{t}^{\prime}
\end{array}\right],
$$

where the sum is over all partitions $\Pi^{\prime}$ satisfying (4).

But in terms of compositions (2), this becomes

$$
\left|\operatorname{fix} \sigma^{2}\right|=c\left(k-1, \frac{m-x}{d}, \frac{n-k x}{d}\right) \cdot\left(\begin{array}{l}
m / d \\
x / d
\end{array}\right) \text {. }
$$

Given $l, d=m / \operatorname{gcd}(l, m)$ divides $\operatorname{gcd}(n, m, x)$, and conversely, given a divisor $d$ of $\operatorname{gcd}(n, m, x)$, there are precisely $\phi(d)$ integers $l$ with $\operatorname{gcd}(2, m)=m / d$. Thus altogether

$$
\sum_{l=1}^{m}\left|\operatorname{fix} \sigma^{l}\right|=\sum_{d} \phi(d) \cdot\left(\begin{array}{l}
m / d \\
x / d
\end{array}\right) c\left(k-1, \frac{m-x}{d}, \frac{n-k x}{d}\right)
$$

where the summation is again over all $d$ such that $d \mid \operatorname{gcd}(n, m, x)$.

(b) Let $g=\rho$. Then $r g=r$ if and only if, for all $i$,

$$
r_{i}=r_{m+1-i} \text {. }
$$

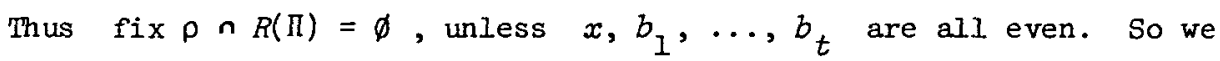
assume that

$$
x=2 x^{\prime \prime}, b_{i}=2 b_{i}^{\prime \prime} \text { for } i=1,2, \ldots, t,
$$

and hence that $\Pi$ corresponds to a partition

$$
\left\{\begin{array}{c}
\Pi^{\prime \prime}=a_{1}^{b_{1}^{\prime \prime}} \cdots a_{t}^{b_{t}^{\prime \prime}} \\
\text { of }(n-x) / 2=\sum_{i=1}^{t} a_{i} b_{i}^{\prime \prime} \text { into }(m-x) / 2=\sum_{i=1}^{t} b_{i}^{\prime \prime} \text { parts, where } \\
1 \leqq a_{1}<a_{2}<\ldots<a_{t} \leqq k-1 \text {, and } t \geqq 1 .
\end{array}\right.
$$

(5)

In this case

$$
\mid \text { fix } \rho \cap R(\Pi) \mid=\left(\begin{array}{c}
m / 2 \\
b_{1}^{\prime \prime}, \ldots, b_{t}^{\prime \prime}, x^{\prime \prime}
\end{array}\right)=\left(\begin{array}{c}
m / 2 \\
x / 2
\end{array}\right) \cdot\left(\begin{array}{c}
(m-x) / 2 \\
b_{1}^{\prime \prime}, \ldots, b_{t}^{\prime \prime}
\end{array}\right) .
$$

Summing over $\Pi$, we have

https://doi.org/10.1017/S000497270002092X Published online by Cambridge University Press 


$$
\mid \text { fix } \rho \mid=\left\{\begin{array}{l}
0 \text { if } \operatorname{gcd}(n, x) \text { is odd, } \\
\left\{\begin{array}{l}
m / 2 \\
x / 2
\end{array}\right) \sum\left[\begin{array}{c}
(m-x) / 2 \\
b_{1}^{\prime \prime}, \ldots, b_{t}^{\prime \prime}
\end{array}\right] \text { if } \operatorname{gcd}(n, x) \text { is even, } \\
\text { where the sum is over all partitions } I^{\prime \prime} \text { satisfying (5). }
\end{array}\right.
$$

In terms of compositions, this becomes

$$
\mid \text { fix } \rho \mid=\left\{\begin{array}{l}
0 \text { if } \operatorname{gcd}(n, x) \text { is odd, } \\
\left(\begin{array}{l}
m / 2 \\
x / 2
\end{array}\right) c\left(k-1, \frac{m-x}{2}, \frac{n-k x}{2}\right) \text { if } \operatorname{gcd}(n, x) \text { is even. }
\end{array}\right.
$$

(c) Let $g=\rho \sigma$. Then $r \rho \sigma=r$ if and only if for all $i=2, \ldots, m, i \neq(m+2) / 2$,

$$
r_{i}=r_{m+2-i}
$$

Again two cases arise:

(i) if $r_{1}=r_{(m+2) / 2}$, then $x, b_{1}, \ldots, b_{t}$ are all even, and the argument of ( $b$ ) above shows that such sequences make a contribution to $|f i x \rho \sigma|$ of

$$
\left\{\begin{array}{l}
0, \text { if } \operatorname{gcd}(n, x) \text { is odd, } \\
\left(\begin{array}{l}
m / 2 \\
x / 2
\end{array}\right) \cdot c\left(k-1, \frac{m-x}{2}, \frac{n-k x}{2}\right) \text { if } \operatorname{gcd}(n, x) \text { is even; }
\end{array}\right.
$$

(ii) if $r_{1} \neq r_{(m+2) / 2}$, then two cases again arise, depending on the parity of $x$.

If $x$ is odd, then one of $r_{1}$ and $r_{(m+2) / 2}$ equals $k$, and the other equals $u$, say where $1 \leqq u \leqq \min (k-1, n-k)$. The rest of the sequence $r$ is determined by the subsequence $\left(r_{2}, \ldots, r_{m / 2}\right)$ which contains $(x-1) / 2$ terms equal to $k$. Its remaining terms constitute a composition of $(n-k x-u) / 2$ into $(m-x-1) / 2$ parts, each part at most $k-1$. In this case the contribution to $\mid$ fix $\rho \circ \mid$ is

$$
\left\{\begin{array}{l}
2, \text { if } m=x+1, \text { so that } u=n-k x<k, \\
2\left(\begin{array}{l}
(m-2) / 2 \\
(x-1) / 2
\end{array}\right) \cdot \sum_{u} c\left(k-1, \frac{m-1-x}{2}, \frac{n-k x-u}{2}\right), \text { if } m>x+1,
\end{array}\right.
$$

where the summation is over all $u$ such that

$$
1 \leqq u \leqq \min (k-1, n-(k-1) x-m+1) \text { and } u \equiv n-k x(\bmod 2) \text {. }
$$

If $x$ is even, then neither $r_{1}$ nor $r_{(m+2) / 2}$ equals $k$; let 


$$
\left\{r_{1}, r_{(m+2) / 2}\right\}=\{u, v\},
$$

where $1 \leqq u<v \leqq k-1$. The rest of the sequence $r$ is determined by the subsequence $\left(r_{2}, \ldots, r_{m / 2}\right)$ which contains $x / 2$ terms equal to $k$. Its remaining terms constitute a composition of $(n-k x-u-v) / 2$ into $(m-x-2) / 2$ parts, each part at most $k-1$. In this case the contribution to $|f i x p o|$ is

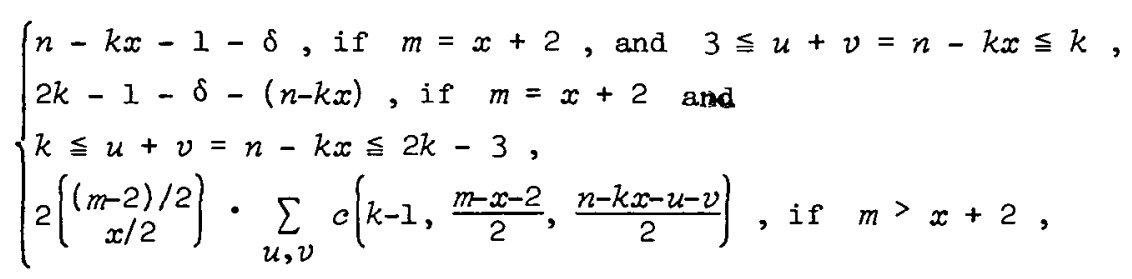

where $\delta=0$ or $1, \delta \equiv n-1(\bmod 2)$, and the summation is over all $u, v$ such that

(7) $\quad 1 \leq u<v \leq k-1, u+v \leq n-(k-1) x-m+2$, and $u+v \equiv n-k x(\bmod 2)$. Note here that the term for $m=x+2$ is simply the number of solutions of

$$
u+v=n-k x,
$$

where $1 \leqq u<v \leqq k-1$. For convenience, we denote this number by $s(m, 2)$.

Cases (a), (b), and (c) cover all possibilities, since $G \backslash(\sigma)$ consists of $m / 2$ elements conjugate to $\rho$, and $m / 2$ elements conjugate to $\rho \sigma$, and conjugate elements fix sets of the same size. Hence we have only to sum the appropriate terms.

First, suppose that $x$ is odd. If $m=x+1$, then $n-k x<k$ and

$$
2 m \cdot F(n, k, m, m-1)=\left(\begin{array}{c}
m \\
m-1
\end{array}\right) \cdot c(k-1,1, n-k x)+m=2 m ;
$$

if $m>x+1$, then

$2 m \cdot F(n, k, m, x)$

$=\sum_{d} \phi(d) \cdot\left(\begin{array}{l}m / d \\ x / d\end{array}\right) \cdot c\left(k-1, \frac{m-x}{d}, \frac{n-k x}{d}\right)+m\left(\begin{array}{l}(m-2) / 2 \\ (x-2) / 2\end{array}\right) \underset{u}{\sum_{u} c\left(k-1, \frac{m-1-x}{2}, \frac{n-k x-u}{2}\right)}$,

where the summations are (respectively) over all $d$ such that

$d \mid \operatorname{gcd}(n, m, x)$ and all $u$ satisfying (6). 
This confirms Theorem 1 (b) (i).

Secondly, suppose that $x$ is even. If $m=x+2$, then $n-k x \leqq 2 k-2$ and

$$
\begin{array}{r}
2 m \cdot F(n, k, m, m-2)=\left(\begin{array}{l}
m \\
2
\end{array}\right) \cdot c(k-1,2, n-k x)+\delta \cdot \frac{m}{2} \cdot c\left(k-1,1, \frac{n-k x}{2}\right) \\
+\delta \cdot m \cdot \frac{m}{2} c\left(k-1,1, \frac{n-k x}{2}\right)+\frac{m}{2} \cdot s(m, 2),
\end{array}
$$

where $\delta=0$ or $1, \delta \equiv n-1(\bmod 2)$.

Now

$$
c(k-1,2, n-k x)=\left\{\begin{array}{l}
n-k x-1, \text { for } n-k x \leqq k, \\
2 k-1-(n-k x), \text { for } k \leqq n-k x \leqq 2 k-2,
\end{array}\right.
$$

and $s(m, 2)=c(k-1,2, n-k x)-\delta$. Hence

$$
2 m \cdot F(n, k, m, m-2)=\frac{m^{2}}{2}\{c(k-1,2, n-k x)+\delta\} .
$$

If $m>x+2$, then

$$
\begin{aligned}
2 m & \cdot F(n, k, m, x)=\sum_{d} \phi(d) \cdot\left(\begin{array}{l}
m / d \\
x / d
\end{array}\right) \cdot c\left(k-1, \frac{m-x}{d}, \frac{n-k x}{d}\right) \\
& +m\left(\begin{array}{c}
(m-2) / 2 \\
x / 2
\end{array}\right) \sum_{u, v} c\left(k-1, \frac{m-x-2}{2}, \frac{n-k x-u-v}{2}\right)+\delta m\left(\begin{array}{l}
m / 2 \\
x / 2
\end{array}\right) \cdot c\left(k-1, \frac{m-x}{2}, \frac{n-k x}{2}\right),
\end{aligned}
$$

where $\delta=0$ or $1, \delta \equiv n-1(\bmod 2)$, and the surmations are (respectively) over all $d$ such that $d \mid \operatorname{gcd}(n, m, x)$ and all $u, v$ satisfying (7). This confirms Theorem $1(b)$.

Note that in either case $(i)$ where $m=x+1$, or (ii) where $m=x+2$, if we use the convention for $c(N, 0, n)$ defined in (3), then the general formula includes these special cases also.

This completes the proof of the theorem.

Proof of Theorem 2 . If $k>n / 2$ and $m \geqq 4$, so that $n \geqq k+3$, then from Theorem 1, we have already that

$$
F(n, k, m, 1)=\frac{1}{2} \cdot c(k-1, m-1, n-k)+\frac{1}{2} \sum_{u} c\left(k-1, \frac{m-2}{2}, \frac{n-k-u}{2}\right),
$$

where the summation is over all $u$ such that $I \leqq u \leqq \min (k-1, n-k-m+2)$ 
and $u \equiv n-k(\bmod 2)$. That is,

$$
F(n, k, m, 1)=\frac{1}{2}\left(\begin{array}{c}
n-k-1 \\
m-2
\end{array}\right)+\frac{1}{2} \sum_{u}\left(\begin{array}{c}
(n-k-u-2) / 2 \\
(m-4) / 2
\end{array}\right) .
$$

Hence if $n / 2<k \leqq n-3$,

$$
F(n, k)=\sum_{m} F(n, k, m, 1)
$$

where the summation is over all even $m$ such that $2 \leqq m \leqq n-k+\varepsilon$, so that

$$
F(n, k)=1+\frac{1}{2} \sum_{m^{\prime}}\left(\begin{array}{c}
n-k-1 \\
2 m^{\prime}
\end{array}\right)+\frac{1}{2} \sum_{u} \sum_{m^{\prime \prime}}\left(\begin{array}{c}
(n-k-u-2) / 2 \\
m^{\prime \prime}
\end{array}\right)
$$

where the summations are (respectively) over all $m^{\prime}$ such that $m=2 m^{\prime}+2$ and $1 \leqq m^{\prime} \leqq[(n-k-1) / 2]$, all $u$ such that

$1 \leqq u \leqq n-k-2$ and $u \equiv n-k(\bmod 2)$, and all $m^{\prime \prime}$ such that

$0 \leqq m^{\prime \prime} \leqq(n-k-u-2) / 2$ and $m=2 m^{\prime \prime}+4 \leqq n-k-u+2$.

Hence by (B1) and (B2),

$$
\begin{aligned}
F(n, k) & =\frac{1}{2}+\frac{1}{2} \cdot 2^{n-k-2}+\frac{1}{2} \sum_{u} 2^{(n-k-u-2) / 2} \text { summing over all } \\
u & \equiv n-k(\bmod 2) \text { such that } 0 \leqq \frac{n-k-u-2}{2} \leqq\left\lfloor\frac{n-k-3}{2}\right\rfloor \\
& =\frac{1}{2}+2^{n-k-3}+\frac{1}{2}(2\lfloor(n-k-1) / 2\rfloor-1) \\
& =2^{n-k-3}+2^{\lfloor(n-k-3) / 2\rfloor} . \quad \square
\end{aligned}
$$

Proof of Theorem 3 . Here we have $n / 3 \leqq k \leqq n / 2$, and $x=1$. Then $k \leqq n-k \leqq 2 k$, and $m \geqq 4$. Also if $m \geqq n-2 k+3$, then

$$
c(k-1, m-1, n-k)=c(m-1, n-k)=\left(\begin{array}{c}
n-k-1 \\
m-2
\end{array}\right) \text {. }
$$

However if $m \leqq n-2 k+2$, then $c(k-1, m-1, n-k)<c(m-1, n-k)$, since we must exclude the compositions of $n-k$ into $m-1$ parts, with one part of size $v, k \leqq v \leqq n-k+m+2$. Each excluded composition corresponds to $m-I$ compositions of $n-k-v$ into $m-2$ parts, so that in this case,

$$
\begin{aligned}
c(k-1, m-1, n-k) & =c(m-1, n-k)-(m-1) \sum_{v} c(m-2, n-k-v) \\
& =\left(\begin{array}{c}
n-k-1 \\
m-2
\end{array}\right)-(m-1) \sum_{v}\left(\begin{array}{c}
n-k-v-1 \\
m-3
\end{array}\right),
\end{aligned}
$$


summing over all $v$ such that $k \leqq v \leqq n-k-m+2$. Hence, from Theorem 1,

$$
\begin{aligned}
& F(n, k,-, 1) \\
& =\frac{1}{2} \sum_{4 \leqq m \leqq n-2 k+2}\left(\left(\begin{array}{c}
n-k-1 \\
m-2
\end{array}\right)-(m-1) \sum_{k \leqq v \leqq n-k-m+2}\left(\begin{array}{c}
n-k-v-1 \\
m-3
\end{array}\right)\right) \\
& +\frac{1}{2} \quad \sum_{m} \quad\left(\begin{array}{c}
n-k-1 \\
m-2
\end{array}\right)+\frac{1}{2} \quad \sum_{m} \sum_{u}\left(\begin{array}{c}
(n-k-u-2) / 2 \\
(m-4) / 2
\end{array}\right) \text {, } \\
& \max (4, n-2 k+3) \leqq m \leqq n-k+\varepsilon \quad 4 \leqq m \leqq n-k+\varepsilon \\
& \text { where } m \text { is even, } \varepsilon=0 \text { or } 1, \varepsilon \equiv n-k(\bmod 2) \text {, and the last } \\
& \text { summation is over all } u \equiv n-k(\bmod 2) \text { such that } \\
& 1 \leqq u \leqq \min (k-1, n-k-m+2) \\
& =\frac{1}{2} \sum_{1 \leqq m^{\prime} \leqq\lfloor(n-k-1) / 2\rfloor}\left(\begin{array}{c}
n-k-1 \\
2 m^{\prime}
\end{array}\right) \\
& -\frac{1}{2} \sum_{4 \leqq m \leqq n-2 k+2}(m-1)\left(\sum_{k \leqq v \leqq n-k-m+2}\left(\begin{array}{c}
n-k-v-1 \\
m-3
\end{array}\right)\right)+\frac{1}{2} \sum_{u}\left(\sum_{m^{\prime \prime}}\left(\begin{array}{c}
(n-k-u-2) / 2 \\
m^{\prime \prime}
\end{array}\right)\right) \\
& \text { where } u \equiv n-k \equiv \varepsilon(\bmod 2), 1 \leqq u \leqq\left\{\begin{array}{l}
k-1, \text { if } n \geqq 2 k+1 \\
k-2, \text { if } n=2 k
\end{array}\right. \text {, } \\
& \text { and } m \text { is even, } m=2 m^{\prime \prime}+4 \text { and } 0 \leqq m^{\prime \prime} \leqq(n-k-u-2) / 2 \text {. }
\end{aligned}
$$

Now

$$
\sum_{k \leqq v \leqq n-k-m+2}\left(\begin{array}{c}
n-k-v-1 \\
m-3
\end{array}\right)=\sum_{m-3 \leqq n-k-v-1 \leqq n-2 k-1}\left(\begin{array}{c}
n-k-v-1 \\
m-3
\end{array}\right)=\left(\begin{array}{c}
n-2 k \\
m-k
\end{array}\right)
$$

by (B3). Hence, by (B4),

(8) $F(n, k,-, 1)$

$$
=\frac{1}{2}\left(2^{n-k-2}-1\right)-\frac{1}{2} \sum_{m}(n-2 k)\left(\begin{array}{c}
n-2 k-1 \\
m-3
\end{array}\right)-\frac{1}{2} \sum_{m}\left(\begin{array}{c}
n-2 k \\
m-2
\end{array}\right)+\frac{1}{2} \sum_{u} 2^{(n-k-u-2) / 2}
$$

surming over all even $m$ such that $4 \leqq m \leqq n-2 k+2$, and all

$$
\begin{gathered}
u \equiv n-k(\bmod 2) \text { such that } 1 \leqq u \leqq\left\{\begin{array}{ll}
k-1, & \text { if } n \geq 2 k+1 \\
k-2, & \text { if } n=2 k
\end{array},\right. \\
=2^{n-k-3}-\frac{1}{2}-\frac{1}{2} \sum_{\mu}(n-2 k)\left(\begin{array}{c}
n-2 k-1 \\
\mu
\end{array}\right)-\frac{1}{2} \sum_{\nu}\left(\begin{array}{c}
n-2 k \\
v
\end{array}\right)+\frac{1}{2} \sum_{j} 2^{j}
\end{gathered}
$$




$$
\begin{aligned}
& \begin{array}{l}
\text { summing over } \mu=m-3 \text {, for } m \text { even and } 1 \leqq \mu \leqq n-2 k-1, \\
\text { over } \nu=m-2 \text {, for } m \text { even and } 2 \leqq \nu \leqq n-2 k \text {, and over } j \text { an } \\
\text { integer, } \frac{n-2 k-1}{2} j \leqq \frac{n-k-3}{3} \\
=2^{n-k-3}-\frac{1}{2}-\frac{1}{2}(n-2 k) 2^{n-2 k-2}-\frac{1}{2}\left(2^{n-2 k-1}-1\right) \\
+\frac{1}{2}\left(2^{\lfloor(n-k-1) / 2\rfloor}-2\lceil(n-2 k-1) / 2\rceil\right) \\
=2^{n-k-3}+2^{\lfloor(n-k-3) / 2\rfloor}-2^{\lceil(n-2 k-3) / 2\rceil}-2^{n-2 k-2}-(n-2 k) 2^{n-2 k-3} .
\end{array}
\end{aligned}
$$

Note that if $n=2 k$ or $2 k+1$, the first and second surmations in equation (8) are vacuous, and the expression reduces to

$$
F(n, k,-, 1)=2^{n-k-3}+2^{\lfloor(n-k-3) / 2\rfloor}-1 .
$$

Proof of Theorem 4. Again $n / 3 \leqq k \leqq n / 2$, but now $x=2$. If $n / 3 \leqq k \leqq n / 2$, and $n \geqq 2 k+4$, then $2 k+4 \leqq n \leqq 3 k$, and for $m \geq 6$ we have (by our remarks preceding the statement of Theorem 4)

$$
\text { (9) } \begin{aligned}
F(n, k, m, 2)=\frac{m-1}{4} \cdot\left(\begin{array}{c}
n-2 k-1 \\
m-3
\end{array}\right) & +\delta \cdot \frac{m+1}{4} \cdot\left(\begin{array}{c}
(n-2 k-2) / 2 \\
(m-4) / 2
\end{array}\right) \\
& +\frac{m-2}{4} \sum_{u, v}\left(\begin{array}{c}
(n-2 k-(u+v)-2) / 2 \\
(m-6) / 2
\end{array}\right) \cdot\left\lfloor\frac{u+v-1}{2}\right\rfloor,
\end{aligned}
$$

where the summation is over all $u, v$ such that $1 \leqq u<v \leqq k-1$, $u+v \equiv n-2 k(\bmod 2)$, and

$$
\frac{m-6}{2} \leqq \frac{n-2 k-(u+v)-2}{2} \leqq\left\lfloor\frac{n-2 k-5}{2}\right\rfloor \text {. }
$$

Consider the last term

$$
L=\frac{m-2}{4} \sum_{u, v}\left(\begin{array}{c}
(n-2 k-(u+v)-2) / 2 \\
(m-6) / 2
\end{array}\right) \cdot\left\lfloor\frac{u+v-1}{2}\right\rfloor .
$$

If $m=6$ and $n=2 k+4$, then $L=0$. If $m=6$ and $n=2 k+5$, then the only possible case is $u=1, v=2$, with $n$ odd, so that

$$
L=\frac{6-2}{4} \cdot\left(\begin{array}{c}
(n-2 k-5) / 2 \\
0 / 2
\end{array}\right) \cdot\left\lfloor\frac{3-1}{2}\right\rfloor=1 \text {. }
$$

Letting $u+v=w$, we deal with the two cases that arise, for $n \geq 2 k+6$.

(i) If $n$ is odd, then $w$ is odd, and

$$
3 \leqq w \leqq n-2 k-m+4,
$$

and 


$$
\frac{w-1}{2}=\frac{n-2 k-1}{2}-\frac{n-2 k-w}{2}
$$

Hence

$$
\begin{aligned}
L & =\sum_{w^{\prime}}\left(\begin{array}{c}
w^{\prime} \\
(m-6) / 2
\end{array}\right)\left(\frac{w-1}{2}\right), \text { where } w^{\prime}=(n-2 k-w-2) / 2 \text { and the summation is over } \\
& \text { all } w^{\prime} \text { such that } \frac{m-6}{2} \leqq w^{\prime} \leqq \frac{n-2 k-5}{2} \\
& =\sum_{w^{\prime}}-\left(w^{\prime}+1\right)\left(\begin{array}{c}
w^{\prime} \\
(m-6) / 2
\end{array}\right)+\frac{n-2 k-1}{2} \sum_{w^{\prime}}\left(\begin{array}{c}
w^{\prime} \\
(m-6) / 2
\end{array}\right) \\
& =-\sum_{w^{\prime}} \frac{m-4}{2}\left(\begin{array}{c}
w^{\prime}+1 \\
(m-4) / 2
\end{array}\right)+\frac{n-2 k-1}{2} \cdot\left(\begin{array}{c}
(n-2 k-3) / 2 \\
(m-4) / 2
\end{array}\right) \\
& =-\frac{m-4}{2} \cdot\left(\begin{array}{c}
(n-2 k-1) / 2 \\
(m-2) / 2
\end{array}\right)+\frac{n-2 k-1}{2}\left(\begin{array}{c}
(n-2 k-3) / 2 \\
(m-4) / 2
\end{array}\right),
\end{aligned}
$$

using the same arguments as in the proof of Theorem 3.

(ii) If $n$ is even, then $w$ is even,

$$
4 \leqq w \leqq n-2 k-n+4 \text {, }
$$

and

$$
\left\lfloor\frac{w-1}{2}\right\rfloor=\frac{w-2}{2}=\frac{n-2 k-2}{2}-\frac{n-2 k-w}{2}
$$

Hence

$$
\begin{aligned}
& L=\sum_{w^{\prime}}\left(\begin{array}{c}
w^{\prime} \\
(m-6) / 2
\end{array}\right)\left(\frac{w-2}{2}\right), \text { where } w^{\prime}=(n-2 k-w-2) / 2, \text { and the summation is } \\
& \text { over all } w^{\prime} \text { such that } \frac{m-6}{2} \leqq w^{\prime} \leqq \frac{n-2 k-6}{2} \\
&=\sum_{w^{\prime}}-\left(w^{\prime}+1\right) \cdot\left(\begin{array}{c}
w^{\prime} \\
(m-6) / 2
\end{array}\right)+\frac{n-2 k-2}{2} \sum_{w^{\prime}}\left(\begin{array}{c}
w^{\prime} \\
(m-6) / 2
\end{array}\right) \\
&=-\sum_{w^{\prime}} \frac{m-4}{2} \cdot\left(\begin{array}{c}
w^{\prime}+1 \\
(m-4) / 2
\end{array}\right)+\frac{n-2 k-2}{2}\left(\begin{array}{c}
(n-2 k-4) / 2 \\
(m-4) / 2
\end{array}\right) \\
&=-\frac{m-4}{2}\left(\begin{array}{c}
(n-2 k-2) / 2 \\
(m-2) / 2
\end{array}\right)+\frac{n-2 k-2}{2}\left(\begin{array}{c}
(n-2 k-4) / 2 \\
(m-4) / 2
\end{array}\right)
\end{aligned}
$$

using the same arguments as in case ( $i$ ) above.

Combining the results of (i) and (ii) with (9) above, we have, for $m \geqq 6$, 


$$
\begin{aligned}
F(n, k, m, 2)=\frac{m-1}{4}\left(\begin{array}{c}
n-2 k-1 \\
m-3
\end{array}\right)+\delta \cdot \frac{m+1}{4}\left(\begin{array}{c}
(n-2 k-2) / 2 \\
(m-4) / 2
\end{array}\right) \\
\quad+\frac{m-2}{4}\left\{\left\lfloor\frac{n-2 k-1}{2}\right]\left(\begin{array}{c}
(n-2 k-3) / 2\rfloor \\
(m-4) / 2
\end{array}\right)-\frac{m-4}{2}\left(\begin{array}{c}
\lfloor(n-2 k-1) / 2\rfloor\}) \\
(m-2) / 2
\end{array}\right)\right\} .
\end{aligned}
$$

For $n \leqq 2 k+5$, so that $m \leqq 6$, we confirm the statement of Theorem 4 immediately.

If $n \geqq 2 k+6$, then

$$
\begin{aligned}
F(n, k,-, 2) & =F(n, k, 4,2)+\sum_{m} F(n, k, m, 2) \\
& =(n-2 k-1+\delta)+\sum F(n, k, m, 2),
\end{aligned}
$$

where the summations are over all even $m$ such that $6 \leqq m \leqq n-2 k+2$. We sum the terms in the second expression:

$$
\text { (a) } \begin{aligned}
\sum_{m} \frac{m-1}{4}\left(\begin{array}{c}
n-2 k-1 \\
m-3
\end{array}\right) & =\frac{1}{4} \sum_{m}\left\{(m-3)\left(\begin{array}{c}
n-2 k-1 \\
m-3
\end{array}\right)+2\left(\begin{array}{c}
n-2 k-1 \\
m-3
\end{array}\right)\right\} \\
& =\frac{1}{4} \sum_{m}\left\{(n-2 k-1)\left(\begin{array}{c}
n-2 k-2 \\
m-4
\end{array}\right)+2\left(\begin{array}{c}
n-2 k-1 \\
m-3
\end{array}\right)\right\} \\
& =\frac{1}{4}(n-2 k-1)\left(2^{n-2 k-3}-1\right)+\frac{1}{2}\left(2^{n-2 k-2}-(n-2 k-1)\right) ;
\end{aligned}
$$

(b) $\sum_{m} \delta \cdot \frac{m+1}{4}\left[\begin{array}{c}(n-2 k-2) / 2 \\ (m-4) / 2\end{array}\right)$

$$
\begin{aligned}
& =\frac{\delta}{2} \sum_{m}\left\{\frac{m-4}{2}\left\{\begin{array}{c}
(n-2 k-2) / 2 \\
(m-4) / 2
\end{array}\right)+\frac{5}{2}\left\{\begin{array}{c}
(n-2 k-2) / 2 \\
(m-4) / 2
\end{array}\right)\right\} \\
& =\frac{\delta}{2} \sum_{m}\left\{\frac{n-2 k-2}{2}\left(\begin{array}{c}
(n-2 k-4) / 2 \\
(m-6) / 2
\end{array}\right)+\frac{5}{2}\left(\begin{array}{c}
(n-2 k-2) / 2 \\
(m-4) / 2
\end{array}\right)\right\} \\
& =\frac{\delta(n-2 k-2)}{4} \cdot 2^{(n-2 k-4) / 2}+\frac{5 \delta}{4} \cdot\left(2^{(n-2 k-2) / 2}-1\right) \\
& =\delta(n-2 k-2) \cdot 2^{(n-2 k-8) / 2}+5 \cdot \delta \cdot 2^{(n-2 k-6) / 2}-\frac{5 \delta}{4} ;
\end{aligned}
$$

(c) $\sum_{m} \frac{m-2}{4} \cdot\left[\frac{n-2 k-1}{2}\right\rfloor \cdot\left(\begin{array}{c}(n-2 k-3-\delta) / 2 \\ (m-4) / 2\end{array}\right)$

$$
\begin{aligned}
& =\frac{1}{2}\left\lfloor\frac{n-2 k-1}{2}\right\rfloor\left\{\sum_{m} \frac{n-2 k-3-\delta}{2}\left(\begin{array}{c}
(n-2 k-5-\delta) / 2 \\
(m-6) / 2
\end{array}\right)+\left(\begin{array}{c}
(n-2 k-3-\delta) / 2 \\
(m-4) / 2
\end{array}\right)\right\} \\
& =\left\{\lfloor(n-2 k-1) / 2\rfloor{ }_{2}\lfloor(n-2 k-5) / 2\rfloor+\frac{1}{2}\left\lfloor\frac{n-2 k-1}{2}\right\rfloor(2\lfloor(n-2 k-3) / 2\rfloor-1) ;\right.
\end{aligned}
$$




$$
\text { (d) } \begin{aligned}
-\sum_{m} \frac{(m-2)(m-4)}{8}\left(\begin{array}{c}
\lfloor(n-2 k-1) / 2\rfloor \\
(m-2) / 2
\end{array}\right) & =-\sum_{m}\left(\begin{array}{c}
\lfloor(n-2 k-1) / 2\rfloor \\
2
\end{array}\right)\left(\begin{array}{c}
\lfloor(n-2 k-5) / 2\rfloor \\
(m-6) / 2
\end{array}\right) \\
& =-\left(\begin{array}{c}
\lfloor(n-2 k-1) / 2\rfloor \\
2
\end{array}\right) 2\lfloor(n-2 k-5) / 2\rfloor .
\end{aligned}
$$

Note that terms in (c) and (d) occur only for $m \leqq n-2 k+1$.

Adding the results of $(a),(b),(c)$, and (d), and substituting in (1l) we have Theorem 4 .

Proof of Theorem 5. If $k=2$, then $n=m+x$ in order to have any sequences possible at all. Hence

$$
F(n, 2,-, x)=F(n, 2, n-x, x),
$$

and we evaluate $F(n, 2, n-x, x)$ from Theorem 1 . If $x$ is odd, then either $n-x=x+1$, and

$$
F(2 x+1,2, x+1, x)=1
$$

or $n-x \geqq x+3$ and

$$
\begin{aligned}
F(n, 2, n-x, x)= & \frac{1}{2(n-x)} \sum_{d \mid \operatorname{gcd}(n, x)} \phi(d)\left(\begin{array}{c}
(n-x) / d \\
x / d
\end{array}\right) c\left(1, \frac{n-2 x}{d}, \frac{n-2 x}{d}\right) \\
& +\frac{1}{2}\left(\begin{array}{c}
(n-x-2) / 2 \\
(x-1) / 2
\end{array}\right) c\left(1, \frac{n-2 x-1}{2}, \frac{n-2 x-1}{2}\right) \\
= & \frac{1}{2(n-x)} \sum_{d \mid \operatorname{gcd}(n, x)} \phi(d)\left(\begin{array}{c}
(n-x) / d \\
x / d
\end{array}\right)+\frac{1}{2}\left(\begin{array}{c}
(n-x-2) / 2 \\
(x-1) / 2
\end{array}\right) .
\end{aligned}
$$

If $x$ is even, then either $n-x=x$, and

$$
F(2 x, 2, x, x)=1
$$

or $n-x=x+2$, and

$$
F(2 x+2,2, x+2, x)=(x+2) / 2
$$

or $n-x \geqq x+4$ and

$$
\begin{aligned}
& F(n, 2, n-x, x)=\frac{1}{2(n-x)} d \sum_{d \mid \operatorname{gcd}(n, x)} \phi(d)\left(\begin{array}{c}
(n-x) / d \\
x / d
\end{array}\right) c\left(1, \frac{n-2 x}{d}, \frac{n-2 x}{d}\right)+\frac{1}{2}\left(\begin{array}{c}
(n-x) / 2 \\
x / 2
\end{array}\right) \cdot c\left(1, \frac{n-2 x}{2}, \frac{n-2 x}{2}\right), \\
& \text { since no choice of } u \text { and } v \text { is possible, } \\
&=\frac{1}{2(n-x)} d \sum_{\operatorname{gcd}(n, x)} \phi(d)\left(\begin{array}{c}
(n-x) / d \\
x / d
\end{array}\right)+\frac{3}{2}\left(\begin{array}{c}
(n-x) / 2 \\
x / 2
\end{array}\right) . \quad 0
\end{aligned}
$$

Proof of Theorem 6. If $k=3$, and $m>x$, then 


$$
\frac{n-x}{2} \leqq m \leqq n-2 x,
$$

and $m$ can take any even value in this range. Again two cases arise, from Theorem 1 .

If $x$ is odd, then either $m=x+1$, so that $n-3 x<3$, and

$$
F(n, 3, m, m-1)=1
$$

or $m \geqq x+3$, and

$$
\begin{aligned}
F(n, 3, m, x)=\frac{1}{2 m} \sum_{d \mid g c d(n, m, x)} \sum_{+\frac{1}{2}\left(\begin{array}{l}
(m-2) / 2 \\
(x-1) / 2
\end{array}\right)} \sum_{\substack{u=1 \text { or } 2 \\
u=n-x(\text { mod2 })}} c\left(2, \frac{m-1-x}{2}, \frac{n-3 x-u}{2}\right) .
\end{aligned}
$$

Now $c(2, M, n)$ is the number of compositions of $n$ into $M$ parts, where each part is 1 or 2 , so

$$
c(2, M, n)= \begin{cases}\left(\begin{array}{c}
M \\
n-M
\end{array}\right) & \text { for } M \leqq n \leqq 2 M, \\
0 & \text { otherwise. }\end{cases}
$$

Hence

$$
\begin{aligned}
& F(n, 3, m, x) \\
& \quad=\frac{1}{2 m} \sum_{d \mid \operatorname{gcd}(n, m, x)} \phi(d)\left(\begin{array}{l}
m / d \\
x / d
\end{array}\right)\left(\begin{array}{c}
(m-x) / d \\
(n-2 x-m) / d
\end{array}\right)+\frac{1}{2}\left(\begin{array}{l}
(m-2) / 2 \\
(x-1) / 2
\end{array}\right)\left(\begin{array}{c}
(m-1-x) / 2 \\
(n-2 x-m+1-u) / 2
\end{array}\right)
\end{aligned}
$$

where $1 \leqq u \leqq 2, u \equiv n-x(\bmod 2)$.

If $x$ is even, then either $m=x+2$, so that $n-3 x \leqq 4$, and

$$
F(n, 3, m, m-2)=m(n-3 x-1+\delta) / 4
$$

or $m \geq x+4$, and

$$
\begin{aligned}
& F(n, 3, m, n)=\frac{1}{2 m} \sum_{d \mid \operatorname{gcd}(n, m, x)} \phi(d) \cdot\left(\begin{array}{l}
m / d \\
x / d
\end{array}\right) c\left(2, \frac{m-x}{d}, \frac{n-3 x}{d}\right) \\
& +\frac{1}{2}\left(\begin{array}{c}
(m-2) / 2 \\
x / 2
\end{array}\right) \sum_{\substack{1 \leq u<v \leqq 2 \\
u+v \equiv n-3 x(\bmod 2)}} c\left(2, \frac{m-x-2}{2}, \frac{n-3 x-u-v}{2}\right)+\frac{1}{2} \delta\left(\begin{array}{l}
m / 2 \\
x / 2
\end{array}\right) c\left(2, \frac{m-x}{2}, \frac{n-3 x}{2}\right),
\end{aligned}
$$

where $\delta=0$ or $1, \delta \equiv n-1(\bmod 2)$. The second summand makes a contribution here only if $u=1, v=2, n \equiv 1(\bmod 2)$. Hence

$$
\begin{aligned}
F(n, 3, m, x)=\frac{1}{2 m} \sum_{\left.d\right|_{\operatorname{gcd}(n, m, x)}} \phi(d) \cdot\left(\begin{array}{l}
m / d \\
x / d
\end{array}\right)\left(\begin{array}{c}
(m-x) / d \\
(n-2 x-m) / d
\end{array}\right) \\
+\frac{1}{2} \varepsilon\left(\begin{array}{c}
(m-2) / 2 \\
x / 2
\end{array}\right)\left(\begin{array}{c}
(m-x-2) / 2 \\
(n-2 x-m-1) / 2
\end{array}\right)+\frac{1}{2} \delta\left(\begin{array}{l}
m / 2 \\
x / 2
\end{array}\right)\left(\begin{array}{c}
(m-x) / 2 \\
(n-m-2 x) / 2
\end{array}\right),
\end{aligned}
$$

where $\delta, \varepsilon=0$ or $1, \delta \equiv n-1(\bmod 2), \varepsilon \equiv n(\bmod 2)$. 


\section{Tables of results}

The following tables list values of $F(n, k,-, x)$ for $n \leqq 20$, $1 \leqq x \leqq 10$. They were calculated using the algorithm of [4] with appropriate sieving and have been checked against the results of the theorems.

Results for other special cases have also been determined using modified sieving algorithms and will appear subsequently.

TABLE 1. $F(n, k,-, 1)$, the number of twills on $n$ harnesses, with maximum float length $k$ which occurs precisely once per period for $n=4, \ldots, 20, k=1, \ldots, n-1$.

\begin{tabular}{|c|c|c|c|c|c|c|c|c|c|c|c|c|c|c|c|c|c|c|}
\hline 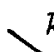 & 1 & 2 & 3 & 4 & 5 & 6 & 7 & 8 & 9 & 10 & 11 & 12 & 13 & 14 & 15 & 16 & 17 & 18 \\
\hline 4 & 0 & & 1 & & & & & & & & & & & & & & & \\
\hline 5 & 0 & & $\bar{I}$ & 1 & & & & & & & & & & & & & & \\
\hline 6 & 0 & 0 & 1 & 1 & 1 & & & & & & & & & & & & & \\
\hline 7 & 0 & 1 & 2 & 2 & 1 & 1 & & & & & & & & & & & & \\
\hline 8 & 0 & 0 & 3 & 2 & 2 & 1 & 1 & & & & & & & & & & & \\
\hline 9 & 0 & 1 & 4 & 5 & 3 & 2 & 1 & 1 & & & & & & & & & & \\
\hline 10 & 0 & 0 & 7 & 7 & 5 & 3 & 2 & 1 & 1 & & & & & & & & & \\
\hline 11 & 0 & 1 & 10 & 14 & 9 & 6 & 3 & 2 & 1 & 1 & & & & & & & & \\
\hline 12 & 0 & 0 & 16 & 22 & 17 & 9 & 6 & 3 & 2 & 1 & 1 & & & & & & & \\
\hline 13 & 0 & 1 & 25 & 43 & 30 & 19 & 10 & 6 & 3 & 2 & $I$ & 1 & & & & & & \\
\hline 14 & 0 & & 40 & 72 & 58 & 33 & 19 & 10 & 6 & 3 & 2 & 1 & 1 & & & & & \\
\hline 15 & 0 & & 62 & 136 & 106 & 66 & 35 & 20 & 10 & 6 & 3 & 2 & 1 & 1 & & & & \\
\hline 16 & 0 & & 101 & 238 & 205 & 122 & 69 & 35 & 20 & 10 & 6 & 3 & 2 & 1 & 1 & & & \\
\hline 17 & 0 & 11 & 159 & 445 & 384 & 242 & 130 & 71 & 36 & 20 & 10 & 6 & 3 & 2 & 1 & 1 & & \\
\hline 18 & 0 & 02 & 257 & 796 & 740 & 460 & 258 & 133 & 71 & 36 & 20 & 10 & 6 & 3 & 2 & 1 & 1 & \\
\hline 19 & 0 & 14 & 410 & 1476 & 1406 & 909 & 498 & 266 & 135 & 72 & 36 & 20. & 10 & 6 & 3 & 2 & 1 & 1 \\
\hline 20 & 0 & 06 & 663 & 2674 & 2710 & 1756 & 988 & 514 & 269 & 135 & 72 & 36 & 20 & 10 & 6 & 3 & 2 & 1 \\
\hline
\end{tabular}


TABLE 2. $F(n, k,-, 2)=$ number of twills of $n$ harnesses with maximum float length $k$ which occurs precisely twice per period for $n=4, \ldots, 20, k=1, \ldots,\lfloor n / 2\rfloor$.

\begin{tabular}{l|rrrrrrrrrr}
\multicolumn{1}{l|}{$k$} & 1 & 2 & 3 & 4 & 5 & 6 & 7 & 8 & 9 & 10 \\
\hline$n$ & 0 & 1 & & & & & & & \\
5 & 0 & 0 & & & & & & & \\
6 & 0 & 2 & 1 & & & & & & \\
7 & 0 & 0 & 0 & & & & & & \\
8 & 0 & 3 & 2 & 1 & & & & & \\
9 & 0 & 0 & 2 & 0 & & & & & \\
10 & 0 & 4 & 5 & 2 & 1 & & & & \\
11 & 0 & 0 & 6 & 2 & 0 & & & & \\
12 & 0 & 5 & 15 & 7 & 2 & 1 & & & \\
13 & 0 & 0 & 18 & 8 & 2 & 0 & & & \\
14 & 0 & 6 & 41 & 23 & 7 & 2 & 1 & & \\
15 & 0 & 0 & 58 & 34 & 10 & 2 & 0 & & \\
16 & 0 & 7 & 113 & 80 & 25 & 7 & 2 & 1 & & \\
17 & 0 & 0 & 174 & 134 & 42 & 10 & 2 & 0 & & \\
18 & 0 & 8 & 325 & 291 & 98 & 27 & 7 & 2 & 1 & \\
19 & 0 & 0 & 514 & 524 & 178 & 44 & 10 & 2 & 0 & \\
20 & 0 & 9 & 929 & 1079 & 392 & 106 & 27 & 7 & 2 & 1
\end{tabular}

TABLE 3. $F(n, k,-, 3)=$ number of twills on $n$ harnesses with maximum float length $k$ which occurs precisely three times per period for $n=4, \ldots, 20, k=1, \ldots,\lfloor(n-1) / 3\rfloor$.

\begin{tabular}{r|rrrrrr}
\multicolumn{1}{c|}{$k$} & 1 & 2 & 3 & 4 & 5 & 6 \\
\hline$n$ & 0 & & & & & \\
5 & 0 & & & & & \\
6 & 0 & & & & & \\
7 & 0 & 0 & & & & \\
8 & 0 & 0 & & & & \\
9 & 0 & 3 & & & & \\
10 & 0 & 0 & 1 & & & \\
11 & 0 & 5 & 1 & & & \\
12 & 0 & 0 & 3 & & & \\
13 & 0 & 8 & 6 & 1 & & \\
14 & 0 & 0 & 11 & 1 & & \\
15 & 0 & 12 & 22 & 4 & & \\
16 & 0 & 0 & 46 & 6 & 1 & \\
17 & 0 & 16 & 82 & 17 & 1 & \\
18 & 0 & 0 & 163 & 32 & 4 & \\
19 & 0 & 21 & 306 & 77 & 7 & 1 \\
20 & 0 & 0 & 572 & 158 & 17 & 1
\end{tabular}


TABLE 4. $F(n, k,-, 4)=$ number of twills on $n$ hamesses with maximum float length $k$, which occurs precisely four times per period, for $n=4, \ldots, 20, k=1, \ldots,|n / 4|$.

\begin{tabular}{|c|c|c|c|c|}
\hline$k$ & 1 & 2 & 3 & 4 \\
\hline 4 & 1 & & & \\
\hline 5 & 0 & & & \\
\hline 6 & 0 & & & \\
\hline 7 & 0 & & & \\
\hline 8 & 0 & 1 & & \\
\hline 9 & 0 & 0 & & \\
\hline 10 & 0 & 3 & & \\
\hline 11 & 0 & 0 & & \\
\hline 12 & 0 & 8 & 1 & \\
\hline 13 & 0 & 0 & 0 & \\
\hline 14 & 0 & 16 & 3 & \\
\hline 15 & 0 & 0 & 3 & \\
\hline 16 & 0 & 29 & 11 & 1 \\
\hline 17 & 0 & 0 & 19 & 0 \\
\hline 18 & 0 & 47 & 49 & 3 \\
\hline 19 & 0 & 0 & 85 & 3 \\
\hline 20 & 0 & 72 & 211 & 14 \\
\hline
\end{tabular}

TABLE 5. $F(n, k,-, 5)=$ number of twills on $n$ harnesses with maximum float length $k$, which occurs precisely five times per period for $n=6, \ldots, 20, k=1, \ldots,\lfloor(n-1) / 5\rfloor$.

\begin{tabular}{r|rrr}
\multicolumn{1}{c|}{$k$} & 1 & 2 & 3 \\
\hline$n$ & 0 & & \\
7 & 0 & & \\
8 & 0 & & \\
9 & 0 & & \\
10 & 0 & & \\
11 & 0 & 1 & \\
12 & 0 & 0 & \\
13 & 0 & 5 & \\
14 & 0 & 0 & \\
15 & 0 & 16 & \\
16 & 0 & 0 & 1 \\
17 & 0 & 38 & 1 \\
18 & 0 & 0 & 5 \\
19 & 0 & 79 & 12 \\
20 & 0 & 0 & 28
\end{tabular}


TABLE 6. $F(n, k,-, 6)=$ number of twills on $n$ harnesses with maximum float length $k$, which occurs precisely six times per period for $n=6, \ldots, 20, k=1, \ldots,\lfloor n / 6\rfloor$.

\begin{tabular}{r|rrr}
\multicolumn{1}{c}{$k$} & 1 & 2 & 3 \\
\hline$n$ & 1 & & \\
7 & 0 & & \\
8 & 0 & & \\
9 & 0 & & \\
10 & 0 & & \\
11 & 0 & & \\
12 & 0 & 1 & \\
13 & 0 & 0 & \\
14 & 0 & 4 & \\
15 & 0 & 0 & \\
16 & 0 & 16 & \\
17 & 0 & 0 & \\
18 & 0 & 50 & 1 \\
19 & 0 & 0 & 0 \\
20 & 0 & 126 & 4
\end{tabular}

TABLE 7. $F(n, k,-, 7)=$ number of twills on $n$ harnesses with maximum float length $k$, which occurs precisely seven times per period for $n=8, \ldots, 20, k=1, \ldots,\lfloor(n-1) / 7\rfloor$.

\begin{tabular}{r|rr}
\multicolumn{1}{c}{$k$} & 1 & 2 \\
\hline 8 & 0 & \\
9 & 0 & \\
10 & 0 & \\
11 & 0 & \\
12 & 0 & \\
13 & 0 & \\
14 & 0 & \\
15 & 0 & 1 \\
16 & 0 & 0 \\
17 & 0 & 8 \\
18 & 0 & 0 \\
19 & 0 & 38 \\
20 & 0 & 0
\end{tabular}


TABLE 8. $F(n, k,-, 8)=$ number of twills on $n$ harnesses with maximum float length $k$, which occurs precisely eight times per period for $n=8, \ldots, 20, k=1, \ldots,\lfloor n / 8\rfloor$.

\begin{tabular}{r|rr}
\multicolumn{1}{c|}{$k$} & 1 & 2 \\
\hline$n$ & 1 & \\
9 & 0 & \\
10 & 0 & \\
11 & 0 & \\
12 & 0 & \\
13 & 0 & \\
14 & 0 & \\
15 & 0 & \\
16 & 0 & 1 \\
17 & 0 & 0 \\
18 & 0 & 5 \\
19 & 0 & 0 \\
20 & 0 & 29
\end{tabular}

TABLE 9. $F(n, k,-, 9)=$ number of twills on $n$ harnesses with maximum float length $k$, which occurs precisely nine times per period for $n=10, \ldots, 20, k=1, \ldots,\lfloor(n-1) / 9\rfloor$.

\begin{tabular}{l|ll}
\multicolumn{1}{c|}{$k$} & 1 & 2 \\
10 & 0 & \\
11 & 0 & \\
12 & 0 & \\
13 & 0 & \\
14 & 0 & \\
15 & 0 & \\
16 & 0 & \\
17 & 0 & \\
18 & 0 & \\
19 & 0 & 1 \\
20 & 0 & 0
\end{tabular}

TABLE 10. $F(n, k,-, 10)=$ number of twills on $n$ harnesses with maximum float length $k$, which occurs precisely ten times per period for $n=10, \ldots, 20, k=1, \ldots,\lfloor(n-1) / 9\rfloor$.

\begin{tabular}{l|ll}
\multicolumn{1}{r}{$k$} & 1 & 2 \\
\hline$n$ & 10 & \\
11 & 0 & \\
12 & 0 & \\
13 & 0 & \\
14 & 0 & \\
15 & 0 & \\
16 & 0 & \\
17 & 0 & \\
18 & 0 & \\
19 & 0 & \\
20 & 0 & 1
\end{tabular}




\section{References}

[1] W. Burnside, Theory of groups of finite order (Cambridge University Press, second edition, 1911; reprinted Dover, London, 1955).

[2] Branko Grünbaum and G.C. Shephard, "Satins and twills; an introduction to the geometry of fabrics", Math. Mag. 53 (1980), 139-161.

[3] Janet A. Hoskins, "Factoring binary matrices: a weaver's approach", Combinatorial Mathematics IX, 300-326 (Lecture Notes in Mathematics, 952. Springer-Verlag, Berlin, Heidelberg, New York, 1982).

[4] W.D. Hoskins and Anne Penfold Street, "Twills on a given number of harnesses", J. Austral. Math. Soc. Ser. A 33 (1982), 1-15.

Departments of Clothing and Textiles, Computer Science, University of Manitoba,

Winnipeg, Manitoba,

Canada R3T 2N2;

Department of Mathematics,

University of Western Australia,

Nedlands,

Western Australia 6009,

Australia;

Department of Mathematics,

University of Queensland,

St Lucia,

Queensland 4067,

Australia. 